\title{
Hilbert Space Structure Induced by Quantum Probes ${ }^{\dagger}$
}

\author{
Go Kato ${ }^{1}$, Masaki Owari ${ }^{2}$ and Koji Maruyama ${ }^{3, *}$ \\ 1 NTT Communication Science Laboratories, NTT Corporation, Atsugi-Shi, Kanagawa 243-0198, Japan; \\ kato.go@lab.ntt.co.jp \\ 2 Department of Computer Science, Shizuoka University, Hamamatsu 432-8011, Japan; \\ masakiowari@inf.shizuoka.ac.jp \\ 3 Department of Chemistry and Materials Science, Osaka City University, Osaka 558-8585, Japan \\ * Correspondence: maruyama@sci.osaka-cu.ac.jp \\ + Presented at the 11th Italian Quantum Information Science conference (IQIS2018), Catania, Italy, \\ 17-20 September 2018.
}

Published: 25 June 2019

\begin{abstract}
It is unrealistic to control all of the degrees of freedom of a high-dimensional quantum system. Here, we consider a scenario where our direct access is restricted to a small subsystem $S$ that is constantly interacting with the rest of the system $E$. What we investigate is the fundamental structures of the Hilbert space and the algebra of hamiltonians that are caused solely by the restrictedness of the direct control. One key finding is that hamiltonians form a Jordan algebra, and this leads to a significant observation that there is a sharp distinction between the cases of $\operatorname{dim} \mathcal{H}_{S} \geq 3$ and $\operatorname{dim} \mathcal{H}_{S}=2$ in terms of the nature of possible operations in $E$. Since our analysis is totally free from specific properties of any physical systems, it would form a solid basis for obtaining deeper insights into quantum control related issues, such as controllability and observability.
\end{abstract}

Keywords: quantum control; Jordan algebra; dynamical Lie algebra

\section{Introduction}

Understanding the dynamics of many-body quantum systems under artificial control is by no means easy. As the race towards the realisation of quantum computer is growing its momentum, a solid theoretical foundation is desired more than ever in order to tame complex quantum dynamics systematically. The principal difficulty is in the necessity of controlling exponentially many degrees of freedom of a large quantum system through a limited number of technically controllable parameters.

Under the common condition of limitations on our artificial control, a natural question would be what we can do on a given physical system, and how it can be done [1-3]. Although it is still somewhat hard to develop methodology of quantum control in a general setting, some useful mathematical results have already been obtained to understand the controllability of a system.

The most noteworthy tool is the dynamical Lie algebra (DLA), which is a set of all realisable operators under the given condition [4-6]. It can be calculated as the maximum set of independent operators that are generated by the (drift) hamiltonian $h_{0}$ and hamiltonians $\left\{h_{k}\right\}$ corresponding to modulable field parameters. In order to make the setting realistic and mathematically tractable, we assume $\left\{h_{k}\right\}$ form a Lie algebra su(dim $\left.\mathcal{H}_{S}\right)$ acting on $\mathcal{H}_{S}$, where $\mathcal{H}_{S}$ is the Hilbert space for a small subsystem $S$ of dimension $\operatorname{dim} \mathcal{H}_{S}$ (Figure 1a). The effects of control on $S$ propagate into the rest of the system, $E$, which interacts with $S$ through $h_{0}$.

Now some questions arise, concerning the DLA under restricted access. What sort of intrinsic constraints are imposed on the algebraic structure of DLA by the restriction on our access? What does the structure of the Hilbert space look like? Does appending an ancillary system $\mathcal{H}_{A}$ to $\mathcal{H}_{S}$ help enlarge the controllable space in $\mathcal{H}_{E}$ ? 
Investigating spatial structures will also have direct and important consequences with respect to the system identifiability. There have been intensive research activities on the problem of quantum system identification under limited access [7-12], and it is becoming clearer that there exist some intrinsic mathematical structures that put limitations on what we can observe through $S$.

(a)

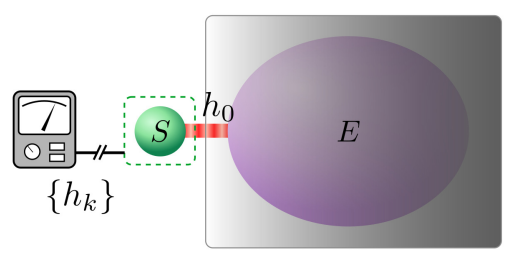

(b)

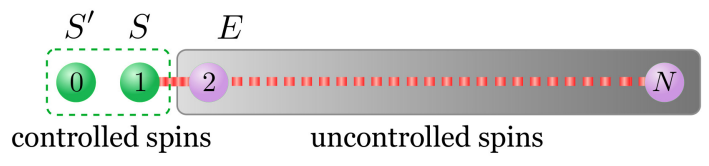

Figure 1. (a) A schematic view of the problem setting. A small subsystem $S$ can be directly accessible, while the rest of the system $E$ is beyond direct control; (b) A one-dimensional spin chain considered for control in [2]. The two spins at the chain end are in the directly accessible subsystem, and the rest of the chain, $E$, only evolves through $h_{0}^{\mathrm{XX}}$.

Here, we classify the algebraic structure of the DLA, induced by the restrictedness of access. In addition, we find that there is a clear distinction between the case of $\operatorname{dim} \mathcal{H}_{S} \geq 3$ and that of $\operatorname{dim} \mathcal{H}_{S}=2$. While there appear only direct sums of $\operatorname{su}(\cdot)$ when $\operatorname{dim} \mathcal{H}_{S} \geq 3$, the structure of formally real Jordan algebra is embedded in the DLA if $\operatorname{dim} \mathcal{H}_{S}=2$. Further, looking into the transition between these two cases, we can answer the question about the effect of ancilla: appending an ancilla does enhance the controllability of quantum state of $E$ if $\operatorname{dim} \mathcal{H}_{S}=2$, although it does not otherwise.

Jordan algebras were first introduced by Jordan et al. more than 80 years ago. Roughly speaking, it is a nonassociative algebra whose multiplication is defined by anticommutator. It has not attracted much attention in quantum mechanics for a long time, however, recently there are some interesting works that discuss Jordan algebraic aspects in topics of quantum spin dynamics (See, e.g., $[13,14]$ ).

\section{Main Results}

The physical setup we consider is as follows. A quantum system $\mathcal{H}_{S}$, on which arbitrary control can be applied at will, is interacting with an external system $\mathcal{H}_{E}$ coherently. The dynamics of $\mathcal{H}_{E}$, including the interaction with $\mathcal{H}_{S}$, is described by the drift Hamiltonian $h_{0}$, and $\mathcal{H}_{E}$ is not subject to our direct control. That is, we can access $\mathcal{H}_{E}$ only indirectly through $\mathcal{H}_{S}$. Also, we assume that the Hilbert spaces $\mathcal{H}_{E}$ and $\mathcal{H}_{S}$ are both finite dimensional.

In the analysis of the controllability of a quantum system, crucially important is the dynamical Lie algebra $L$. It is a Lie algebra generated by $i h_{0}$ and a set $\left\{I d_{E}\right\} \otimes \operatorname{su}\left(\operatorname{dim} \mathcal{H}_{S}\right)$ of operators. Here, $I d_{E}$ is the identity operator on $\mathcal{H}_{E},\left\{I d_{E}\right\}$ is a one-dimensional space generated by $I d_{E}$, and $\operatorname{su}\left(\operatorname{dim} \mathcal{H}_{S}\right)$ is a set of all traceless skew-hermitian operators acting on $\mathcal{H}_{S}$, thus representing a set of arbitrary controls. A direct product of the operator sets $\mathcal{S}_{1} \otimes \mathcal{S}_{2}$ is a set of $s_{1} \otimes s_{2}$ for all $s_{b} \in \mathcal{S}_{b}(b=\{1,2\})$, and $i \mathcal{S}$ is the set of elements $i \cdot s$ for all $s \in \mathcal{S}$.

Before presenting our main results, let us define two terms. One is the connected algebra $L_{c}$, which is the smallest ideal of $L$ that includes $\left\{I d_{E}\right\} \otimes \operatorname{su}\left(\operatorname{dim} \mathcal{H}_{S}\right)$, and the other is the disconnected algebra $L_{d}$; it is a set of all skew-hermitian operators which commute with any element in $L_{c}$. Formally,

$$
\begin{aligned}
& L_{c}:=\mathcal{L}\left(\left\{\left[\cdots\left[\left[g^{\prime}, g_{1}\right], g_{2}\right], \cdots, g_{n}\right] \mid n \in \mathbb{Z}_{\geq 1} \wedge g_{m} \in L \wedge g^{\prime} \in\left\{I d_{E}\right\} \otimes \operatorname{su}\left(\operatorname{dim} \mathcal{H}_{S}\right)\right\}\right), \\
& L_{d}:=\left\{g \mid g \in \mathbf{u}\left(\operatorname{dim} \mathcal{H}_{E} \cdot \operatorname{dim} \mathcal{H}_{S}\right) \wedge \forall g^{\prime} \in L_{c},\left[g, g^{\prime}\right]=0\right\}
\end{aligned}
$$

where $\mathcal{L}(\mathcal{S})$ indicates a set of all real linear combinations of the elements in $\mathcal{S}$, and $\mathrm{u}\left(\operatorname{dim} \mathcal{H}_{E} \cdot \operatorname{dim} \mathcal{H}_{S}\right)$ is a set of all skew-hermitian operators on $\mathcal{H}_{E} \otimes \mathcal{H}_{S}$. From the Jacobi relation, $L_{d}$ is also a Lie algebra.

We list here the rough and intuitive messages of the five main theorems on the structure of the DLA induced by the restrictedness of direct access. The precise statement can be found in [15]. 
Theorem 1. Any element in the dynamical Lie algebra $L$ is a sum of two elements, one of which is controllable from operations on $S$ and the other is uncontrollable. These two are the elements of subalgebras $L_{c}$ and $L_{d}$, respectively.

Theorem 2. When $\operatorname{dim} \mathcal{H}_{S} \geq 3$, the Hilbert space $\mathcal{H}_{E}$ can have a direct sum structure:

$$
\mathcal{H}_{E}=\bigoplus_{j} \mathcal{H}_{E_{j}}=\bigoplus_{j} \mathcal{H}_{B_{j}} \otimes \mathcal{H}_{R_{j}}
$$

In accordance with the Decomposition (3), $L_{d}$ and $L_{c}$ are written as direct sums of subalgebras as

$$
L_{d}=\bigoplus_{j} \mathrm{u}\left(\operatorname{dim} \mathcal{H}_{B_{j}}\right) \otimes\left\{I d_{R_{j}} \otimes I d_{S}\right\}, \text { and } L_{c}=\bigoplus_{j}\left\{I d_{B_{j}}\right\} \otimes \mathrm{su}\left(\operatorname{dim} \mathcal{H}_{R_{j}} \cdot \operatorname{dim} \mathcal{H}_{S}\right) .
$$

The dynamics on $\mathcal{H}_{R}$ is driven by $L_{c}$, and that on $\mathcal{H}_{B}$ is by $L_{d}$. Thus, $\mathcal{H}_{B}$ cannot be controlled through operations on $\mathcal{H}_{S}$. In other words, the limitedness of direct access to $S$ induces a natural basis structure to $E$.

Theorem 2 conveys a somewhat strong message. It claims that, when $\operatorname{dim} \mathcal{H}_{S} \geq 3$, even if we attach an additional quantum system $S^{\prime}$ to $S$, intending to enlarge the effective work space, it does not expand the set of executable operations for $\mathcal{H}_{E}$.

Theorem 3. When $\operatorname{dim} \mathcal{H}_{S}=2, \mathcal{H}_{E}$ has a direct sum structure, which is similar to the case of dim $\mathcal{H}_{S} \geq 3$, while the connected dynamical Lie algebra $L_{c}$ may not span su, i.e., the full controllability is not guaranteed.

One common message from Theorems 2 and 3 is that, regardless of the dimension of the system $S$, the system $E$ would have a direct sum structure as in Equation (3). Thus the quantum dynamics cannot make a state jump between different subspaces in the sum.

The proofs of Theorems 1-3 are somewhat intricate, thus let us delineate the basic idea only briefly here. Let $G^{(1)}$ be a set of operators on $\mathcal{H}_{E}$ that form interaction operators with the $S$ system in $L$. Namely, any operator $g_{1}$ in $G^{(1)}$ is in $L$ in the form $g_{1} \otimes \sigma_{S}$ with $\sigma_{S} \in i \cdot \operatorname{su}\left(\operatorname{dim} \mathcal{H}_{S}\right)$. Another one, $G^{(0)}$, is defined to be a set of those operators $g_{0}$ that form $g_{0} \otimes I d_{S}\left(G^{(0)}\right.$ and $G^{(1)}$ are not exclusive). Then, $i\left\{G^{(1)}, G^{(1)}\right\} \subseteq G^{(1)}$ holds regardless of $\operatorname{dim} \mathcal{H}_{S}$, which means that $G^{(1)}$ forms a Jordan algebra [16]. A (formally real) Jordan algebra $J$ is known to be written as a direct sum of simple Jordan algebras $J_{j}$, and this is why our $\mathcal{H}_{E}$ should have a direct sum structure as well. The distinction between the cases of $\operatorname{dim} \mathcal{H}_{S} \geq 3$ and $=2$ comes from another algebraic structure, that is, $\left[G^{(1)}, G^{(1)}\right] \subseteq G^{(1)}$ holds when $\operatorname{dim} \mathcal{H}_{S} \geq 3$, but it does not if $\operatorname{dim} \mathcal{H}_{S}=2$.

Theorem 4. The algebraic structures shown in Theorems 2 and 3 are sufficient conditions for $L$ to be a Lie algebra that contains $\operatorname{su}\left(\operatorname{dim} \mathcal{H}_{S}\right)$.

Theorem 5. This theorem shows how the space structure changes when an additional dimension(s) is appended to a 2-dim $\mathcal{H}_{S}$, thus describes how controllability is acquired in the transition from dim $\mathcal{H}_{S}=2$ to 3.

The expansion of controllable space can be seen in the existing study of quantum controllability of specific physical systems. For example, in [2], the indirect control was discussed for a chain of $N$ spin-1/2 particles whose dynamics is governed by the drift hamiltonian

$$
i h_{0}^{\mathrm{XX}}=\frac{i}{2} \sum_{k=1}^{N} c_{k}\left[(1+\gamma) X_{k} X_{k+1}+(1-\gamma) Y_{k} Y_{k+1}\right]+b_{k} Z_{k}
$$

where the last term represents the Zeeman interaction and $\gamma$ is the anisotropy parameter.

The paper [2] presented a specific and efficient scheme to control the entire chain through $S$ containing two end spins under the dynamics by $i h_{0}^{X X}$ (See Figure $1 b$ ). The inclusion of two spins 
in $S$ is necessary, since directly controlling only one spin at the chain end does not lead to the full controllability over the entire chain. Our results give a clear explanation on the structural difference between these two cases, and what happens when an extra spin, the 0th spin in Figure 1b, is appended.

\section{Conclusions}

We have clarified the structures of the Hilbert space and the Lie algebra that are induced when active controls are possible only in a small subspace of a quantum system. The present analysis can be applied to the study of physical situations where we wish to control a large quantum system with minimal access. Such scenarios have been discussed under the motivation to suppress unnecessary interactions between the quantum system and its environment.

The presented results can be useful to further investigate the possibility of indirect control of large systems. In this context, one significant consequence of indirect control is the existence of equivalence classes, within which any distinct physical configurations of $E$ and its hamiltonians cannot be distinguished by any operations on $S$. While it has already been studied in the literature, such as $[12,17]$, our results would shed more lights on this issue in a consistent way.

Acknowledgments: We acknowledge financial support by the JSPS Kakenhi (C) No. 26400400 and No. 17 K05591. MO also thanks for support by the JSPS Kakenhi (C) No. 16K00014.

\section{References}

1. Lloyd, S.; Landahl, A.J.; Slotine, J.-J.E. Universal quantum interfaces. Phys. Rev. A 2004, 69, 012305, doi:10.1103/PhysRevA.69.012305.

2. Burgarth, D.; Maruyama, K.; Murphy, M.; Montangero, S.; Calarco, T.; Nori, F.; Plenio, M.B. Scalable quantum computation via local control of only two qubits. Phys. Rev. A 2010, 81, 040303(R), doi:10.1103/PhysRevA.81.040303.

3. Kay, A.; Pemberton-Ross, P.J. Computation on spin chains with limited access. Phys. Rev. A 2010, 81, 010301(R), doi:10.1103/PhysRevA.81.010301.

4. Jurdjević, V.; Sussmann, H. Control systems on Lie groups. J. Diff. Eqs. 1972, 12, 313-329.

5. D'Alessandro, D. Introduction to Quantum Control and Dynamics; Taylor and Francis: Boca Raton, FL, USA, 2008.

6. Maruyama, K.; Burgarth, D. Gateway schemes of quantum control for spin networks. In Electron Spin Resonance (ESR) Based Quantum Computing; Takui, T., Berliner, L., Hanson, G., Eds.; Springer: New York, NY, USA, 2016; pp. 167-192.

7. Burgarth, D.; Maruyama, K.; Nori, F. Coupling strength estimation for spin chains despite restricted access. Phys. Rev. A 2009, 79, 020305(R), doi:10.1103/PhysRevA.79.020305.

8. Di Franco, C.; Paternostro, M.; Kim, M.S. Hamiltonian tomography in an access-limited setting without state initialization. Phys. Rev. Lett. 2009, 102, 187203, doi:10.1103/PhysRevLett.102.187203.

9. Burgarth, D.; Maruyama, K. Indirect Hamiltonian identification through a small gateway. New J. Phys. 2009, 11, 103019, doi:10.1088/1367-2630/11/10/103019.

10. Burgarth, D.; Maruyama, K.; Nori, F. Indirect quantum tomography of quadratic Hamiltonians. New J. Phys. 2011, 13, 013019, doi:10.1088/1367-2630/13/1/013019.

11. Shabani, A.; Kosut, R.L.; Mohseni, M.; Rabitz, H.; Broome, M.A.; Almeida, M.P.; Fedrizzi, A.; White, A.G. Efficient measurement of quantum dynamics via compressive sensing. Phys. Rev. Lett. 2011, 106, 100401, doi:10.1103/PhysRevLett.106.100401.

12. Owari, M.; Maruyama, K.; Takui, T.; Kato, G. Probing an untouchable environment for its identification and control. Phys. Rev. A 2015, 91, 012343, doi:10.1103/PhysRevA.91.012343.

13. Zhang, Z.D. Mathematical structure of the three-dimensional(3D) Ising model. Chin. Phys. B 2013, 22, 030513.

14. Ławrynowicz, J.; Nowak-Kepczyk, M.; Suzuki, O. Fractals and chaos related to Ising-Onsager-Zhang lattices versus the Jordan-von Neumann-Wigner procedures. Quaternary approach. Int. J. Bifurc. Chaos 2012, 22, 1230003, doi:10.1142/S0218127412300030. 
15. Kato, G.; Owari, M.; Maruyama, K. Hilbert space structure induced by quantum probes. arXiv 2018, arXiv:1803.11128.

16. Jordan, P.; von Neumann, J.; Wigner, E. On an Algebraic Generalization of the Quantum Mechanical Formalism. Ann. Math. 1934, 35, 29-64.

17. Burgarth, D.; Yuasa, K. Quantum system identification. Phys. Rev. Lett. 2012, 108, 080502, doi:10.1103/PhysRevLett.108.080502.

(C) 2019 by the authors. Licensee MDPI, Basel, Switzerland. This article is an open access article distributed under the terms and conditions of the Creative Commons Attribution (CC BY) license (http:/ / creativecommons.org/licenses/by/4.0/). 